\title{
Image watermarking using discrete wavelet-tchebichef transform
}

\author{
Andik Setyono, De Rosal Ignatius Moses Setiadi
}

Department of Informatics Engineering, Dian Nuswantoro University, Indonesia

\begin{tabular}{|c|c|}
\hline Article Info & ABSTRACT \\
\hline Article history: & \multirow{10}{*}{$\begin{array}{l}\text { Image watermarking is one of the most popular techniques for authenticating } \\
\text { copyright on the digital image. Many research on image watermarking has } \\
\text { proved that the joint of Discrete Cosine Transform (DCT) and Discrete } \\
\text { Wavelet Transform (DWT) combinations can improve both imperceptibility } \\
\text { and robustness when compared to DCT or DWT only. Discrete Tchebichef } \\
\text { Transform (DTT) denotes an alternative transformation that has a similarity } \\
\text { property with DCT. DTT has an advantage in reducing memory requirements } \\
\text { during computing, so the calculation speed is much faster than DCT. This } \\
\text { study tested the performance of DTT and DCT on non-blind image } \\
\text { watermarking method, where DTT and DCT are performed after DWT. } \\
\text { Based on the experimental results, this research proved that the DTT was } \\
\text { combined successfully with DWT and very potential for further investigation } \\
\text { because it has a computing performance much better than DCT. While the } \\
\text { image watermarking quality, both the imperceptibility and robustness aspects } \\
\text { were completely identical with the combination of DCT and DWT } \\
\text { transformation. }\end{array}$} \\
\hline Received Dec 20, 2018 & \\
\hline Revised Mar 17, 2019 & \\
\hline Accepted Apr 11, 2019 & \\
\hline Keywords: & \\
\hline Copyright protection & \\
\hline Fast computation & \\
\hline Reduced memory & \\
\hline Tchebichef & \\
\hline Wavelet & \\
\hline
\end{tabular}

Copyright @ 2019 Institute of Advanced Engineering and Science. All rights reserved.

\section{Corresponding Author:}

De Rosal Ignatius Moses Setiadi,

Department of Informatics Engineering, Dian Nuswantoro University,

Imam Bonjol Street no 207, Semarang 50131, Indonesia.

Email: moses@dsn.dinus.ac.id

\section{INTRODUCTION}

Internet technology allows the spread of data becomes faster and easier. This is certainly very useful for human life. The growing number of internet users, increasing the risk of digital crime. Rules that ensure data security of internet users cannot be guaranteed by current law. This is due to the laws applicable in each different country, while the internet is used by all people in the world [1-3]. Then, it will be needed a method to improve security in transaction data on the internet, especially about copyright protection. Image watermarking is a popular method for authenticating copyright on digital image [4-6]. There are two important aspects of the image watermarking, i.e., imperceptibility and robustness. Imperceptibility is the quality of copyright insertion in the digital images, the better imperceptibility of the human vision cannot perceive changes in the digital images. While robustness is the aspect of copyright endurance against attacks. If the robustness aspect works well, copyright can survive when a digital image is manipulated [7-10].

Implementation of the frequency domain in image watermarking method has proved that more resistant to various attacks compared to spatial domains. The most commonly used frequency domain in image watermarking research is DCT and DWT [11-14]. In the image watermarking method, the combination of DCT and DWT have proved that the method can generate better results than the use of one transformation only [15-18]. This is caused by each transformation has its own advantages. Both of the transformations have also been widely used in various studies on image processing, such as image watermarking, image compression, image restoration, etc. Even has also been embedded in various hardware 
because both transformations are used as JPEG and JPEG 2000 compression standards [16, 19, 20]. DTT is a transformation that can be used as an alternative to DCT. DTT has an advantage in reducing code complexity and memory requirements for computation, thus speeding up the transformation calculation process [21-23], besides many similarities property with the DCT $[9,24]$. DTT can also be implemented in image watermarking [25]. DTT is not a new algorithm as well as DCT, it is just that the use of DTT in watermarking on images is not as popular as DCT. The literature on DTT for image watermarking is also quite limited since DTT is mostly applied to image compression and digital video [21-23, 26, 27]. In Setiadi et al's study [9] there have also been comparisons between DCT and DTT. Aspects of imperceptibility and robustness are also very similar. Surely this research needs to be continued and piloted by combining with other transformations such as Wavelet.

\section{RESEARCH METHOD}

\subsection{Discrete Tchebichef Transform (DTT)}

DTT is one of the derivatives of the orthonormal Tchebichef polynomial [9, 28, 29]. DTT transforms the image with a polynomial recursive technique $r_{q}(d)$. To perform the transformation on image matrix with size $M * M$ where $M=8$ can be seen in (1).

$$
T_{q w}=\sum_{d=0}^{M-1} \sum_{e=0}^{M-1} r_{q}(d) r_{w}(e) f(d, e)
$$

where,

$T \quad:$ is the result of the transformation

$f \quad$ : is the image pixel designated coordinates $d$ and $e$

$q, w$ : recursive order

while the recursive value of the polynomial $r_{q}(d)$ is defined in (2), (3), and (4).

$$
\begin{aligned}
& r_{0}(d)=\frac{1}{\sqrt{M}} \\
& r_{1}(d)=(2 d+1-M) \sqrt{\frac{3}{M\left(M^{2}-1\right)}} \\
& r_{q}(d)=\left(A_{1} d+A_{2}\right) r_{q-1}(d)+A_{3} r_{q-2}(d),
\end{aligned}
$$

where: $q=2, \ldots M-1$

To calculate the coefficients $A_{1}, A_{2}, A_{3}$, use (5), (6), and (7).

$$
\begin{aligned}
& A_{1}=\frac{2}{q} \sqrt{\frac{4 q^{2}-1}{M^{2}-q^{2}}} \\
& A_{2}=\frac{1-M}{q} \sqrt{\frac{4 q-1}{M^{2}-q^{2}}} \\
& A_{3}=\frac{q-1}{q} \sqrt{\frac{2 q-1}{2 q-3}} \sqrt{\frac{M^{2}-(q-1)^{2}}{M^{2}-q^{2}}}
\end{aligned}
$$

while to perform inverse transformation DTT use (8).

$$
f_{(d, e)}=\sum_{q=0}^{M-1} \sum_{w=0}^{M-1} T_{q w} r_{q}(d) r_{w}(e)
$$

\subsection{Similarities of DTT and DCT Proposed Method}

Calculations of DTT and DCT have similarities, the first is a separable calculation. DTT and DCT can be written separately to calculate one-dimensional data, by applying the same way applicable to twodimensional data. Second is the calculation of DTT and DCT can be symmetrically done. The third is DTT and DCT using orthogonal basis. Where in the lowest frequency base image there will be a top left corner or coordinates $(0,0)$ and the highest frequency is in the lower left corner or coordinates $(7,7)[21,26,27,29]$. Figure 1 shows the DCT and DTT two-dimensional image base. Another DTT and DCT similarities are having the same energy compaction way, that is, the core energy of the packed and compressed image to the 
low-frequency region. The compaction properties of the energy are very good for the highly correlated image so that the distortion that occurs during the reconstruction process is relatively small.

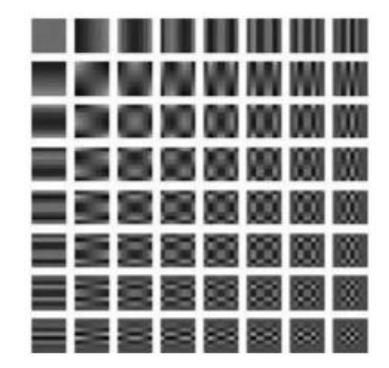

(a)

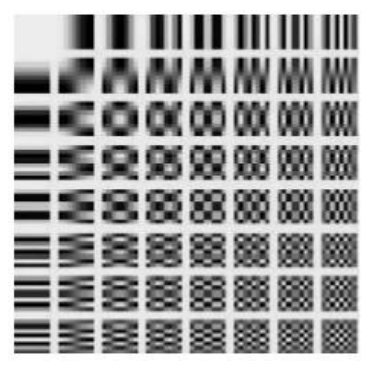

(b)

Figure 1. 2-D image base of (a) DTT, (b) DCT

\subsection{Discrete Wavelet Transform (DWT)}

DWT is used as a transformation of the JPEG 2000 compression standard. Unlike DCT and DTT which transform images in high, medium and low frequencies. DWT divides the image into four subbands, ie LL, HL, LH, HH [12, 30, 31]. Where each subband contains a collection of frequencies. This subband division uses two kinds of filters that are low pass filter and high pass filter that is imposed on a row and column image. Subband LL contains low-frequency imagery, where image energy is centered here. The HL and LH subband contains the frequency of horizontal and vertical image approximation results. Whereas $\mathrm{HH}$ subband contains a high-frequency image or diagonal image. Figure 2 describes the result of image transformation with DWT.

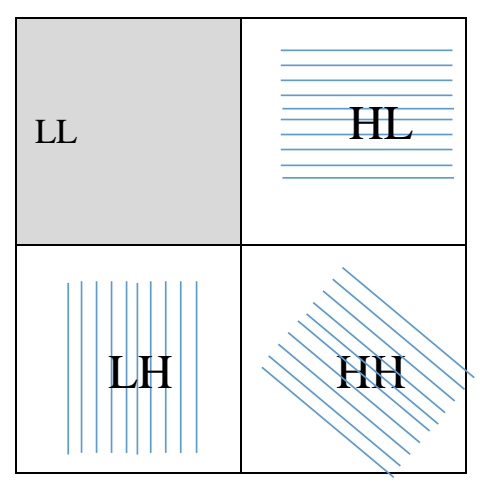

Figure 2. 2-D discrete wavelet transform

\subsection{Proposed Watermark Insertion Scheme}

The watermark insertion scheme used in this study is to combine DWT and DTT. Because in this research DTT is compared with DCT then the watermark insertion process is done by the same step between DTT and DCT, along with the alpha value used. Figure 3 shows a clear description of the steps of inserting a watermarking process. The steps below are the details of the process of insertion watermark proposed.

Step 1: Read the host image, then do DWT on the host image

Step 2: Select the LL subband, then divide the subband into a small sub-blocks of size $4 * 4$

Step 3: Transform each sub-blocks using DTT.

Step 4: Collect coefficient $(1,1)$.

Step 5: Read the watermark image

Step 6: Insert watermark image into collected coefficient $(1,1)$ using the alpha value

Step 7: Do inverse DTT, then inverse DWT, and get the watermarked image. 


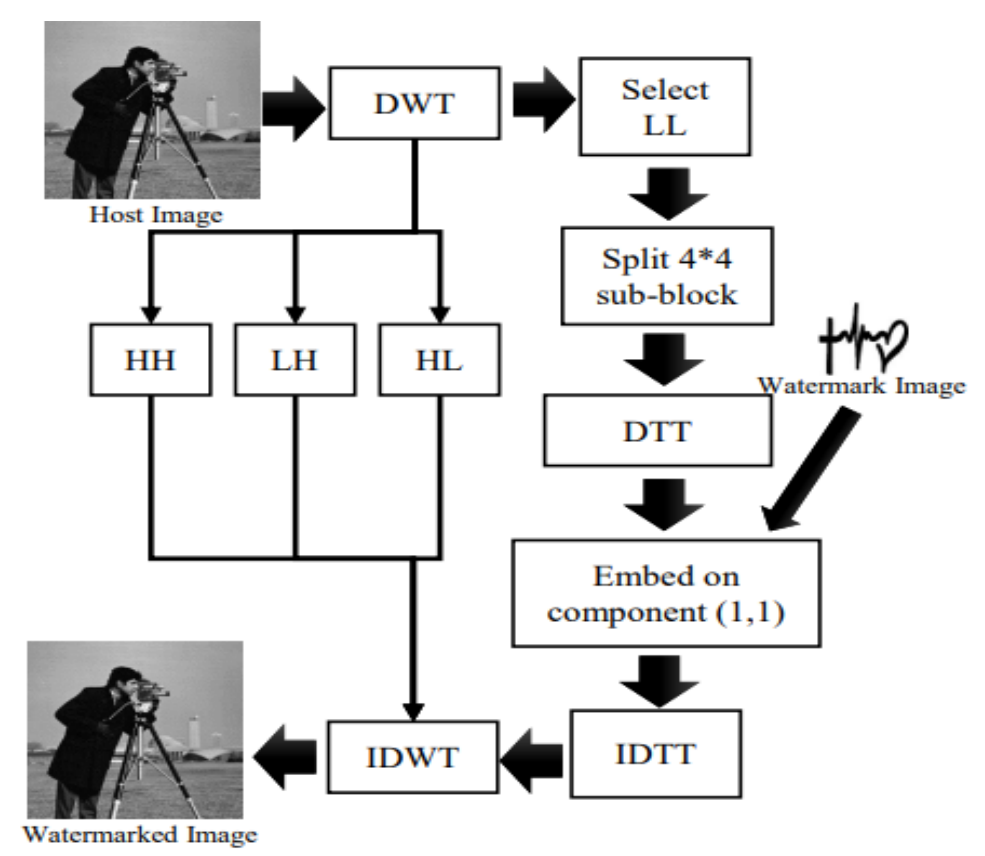

Figure 3. Watermark insertion scheme

\subsection{Watermark Extraction Scheme}

As the insertion process, the extraction process is also carried out with the same steps for DTT and DCT. Both transformations are done after DWT. Figure 4 shows a description of the steps of the watermark extraction process. The steps below are the details of the process of extraction watermark proposed.

Step 1 : Read the host image also watermarked image, then do DWT on both images.

Step 2 : Select the LL subbands on each image.

Step 3 : Divide the LL subband into a small sub-blocks with size $4 * 4$.

Step 4 : Transform each sub-blocks using DTT.

Step 5 : Collect the coefficient $(1,1)$ and save it on a different matrix.

Step 6 : Perform the extraction process by comparing the coefficient matrix $(1,1)$ in the host image and the watermarked image using the alpha value.

Step 7 : Get the watermark image.

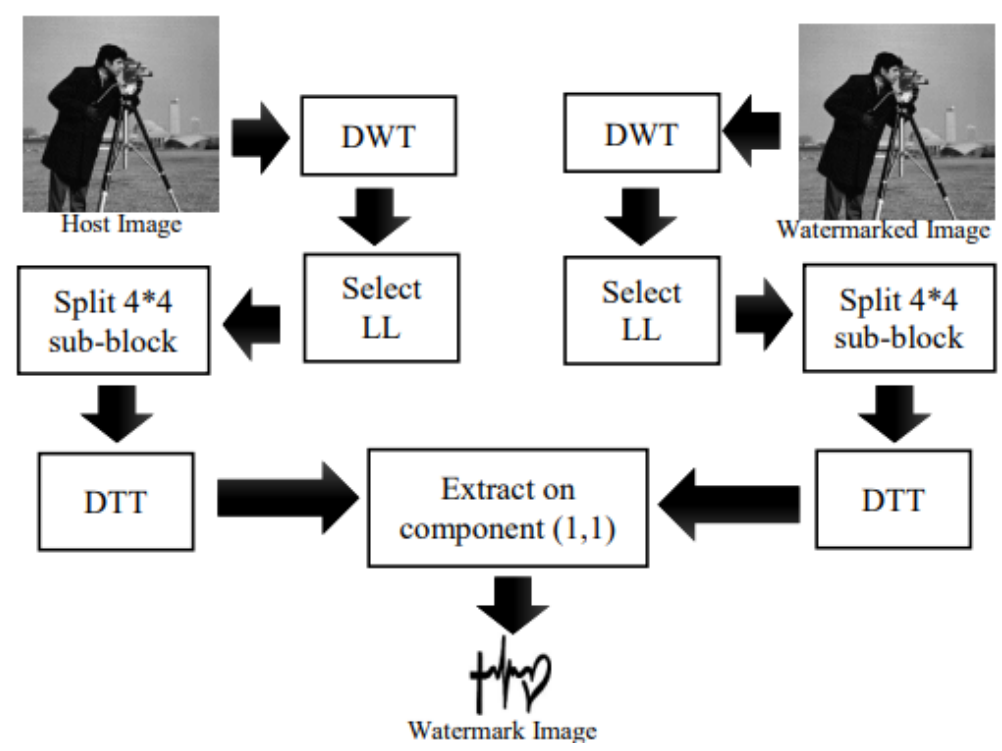

Figure 4. Watermark extraction scheme 


\section{RESULTS AND ANALYSIS}

This section begins with collecting image data used in the test. The image used as the host image is the grayscale image and the binary image for the watermark. There are seven cover images used with $512 *$ 512. While the watermark image size is $64 * 64$. The image used is a standard image that is widely used in various studies of image processing, this is to facilitate comparison for subsequent research. Furthermore, the watermark image is inserted with the proposed method for a comparative test. Figure 5 displays the image used in this study.

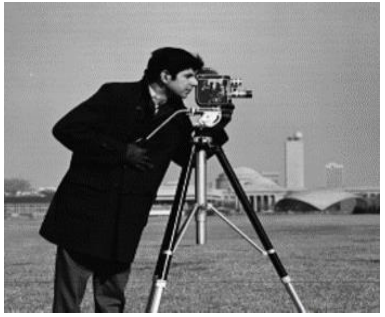

(a)

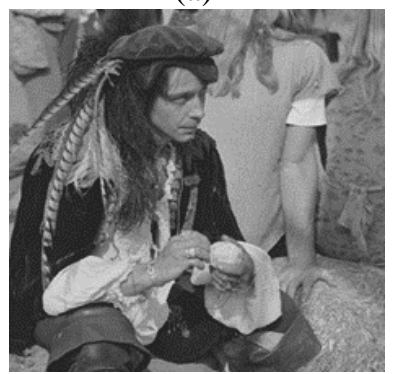

(e)

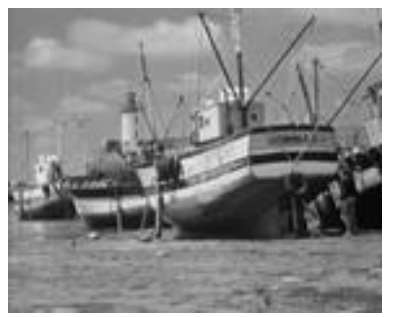

(b)

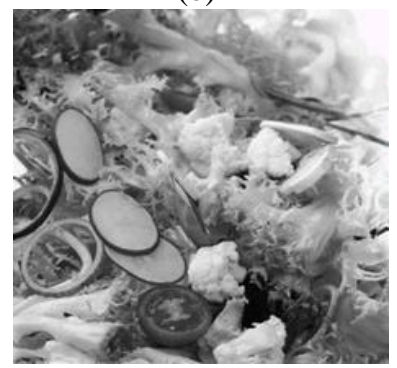

(f)

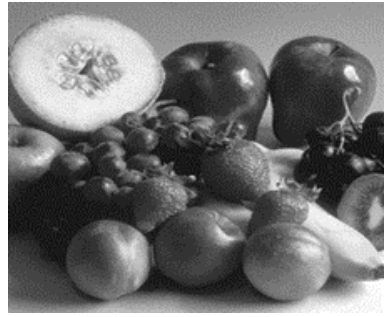

(c)

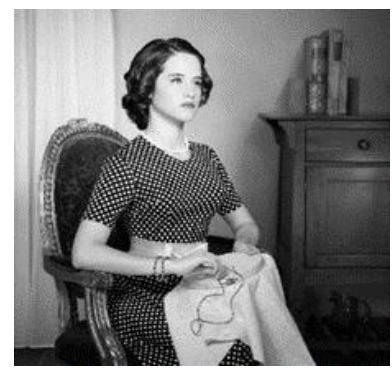

(g)

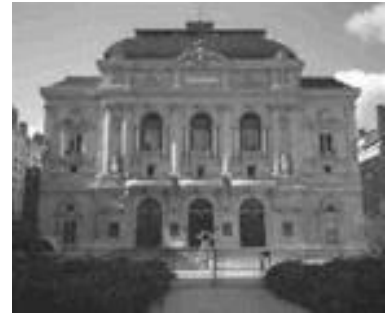

(d)

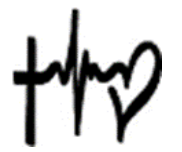

(h)

Figure 5. (a-g) Cover image used \{(a) cameraman; (b) fishing boat; (c) fruit; (d) opera; (e) pirate; (f) vegetable; (g) women \}; (h) watermark image

This study evaluated DTT-DWT combination performance when applied to non-blind watermarking techniques. The DTT-DWT combination is also comparable to the DCT-DWT combination. Both combinations of these methods will be similarly measured for performance in the watermark insertion and extraction scheme. Figure 6 shows the sample image of the watermarked insertion with the proposed method. It can be seen in Figure 6 by plain view no difference at all between the watermark image with the DWTDTT method, DWT-DCT method, and with the original image. So this study uses MSE and PSNR to measure the imperceptibility quality of the watermarked image. Where the measurement of MSE value using (9) while the PSNR value using (10) [32, 33]. Table 1 shows the measurements of MSE and PSNR values.

$$
\begin{aligned}
& M S E=\sum_{m=0}^{M-1} \sum_{n=0}^{N-1}\|W(m, n)-H(m, n)\|^{2} \\
& P S N R_{d B}=10 \log 10\left(\frac{255^{2}}{\sqrt{M S E}}\right)
\end{aligned}
$$

where $m$ and $n$ is the size of row and column image, $W$ is watermarked image and $H$ is host image.

Table 1. Comparative MSE and PSNR values

\begin{tabular}{ccccc}
\hline \multirow{2}{*}{ Image } & \multicolumn{2}{c}{ DWT-DTT } & \multicolumn{2}{c}{ DWT-DCT } \\
& MSE & PSNR & MSE & PSNR \\
\hline cameraman & 12.2867 & 37.2365 & 12.2867 & 37.2365 \\
fishingboat & 12.2888 & 37.2357 & 12.2888 & 37.2357 \\
fruit & 12.2305 & 37.2564 & 12.2305 & 37.2564 \\
opera & 12.2575 & 37.2468 & 12.2575 & 37.2468 \\
pirate & 12.2891 & 37.2356 & 12.2891 & 37.2356 \\
vegetable & 12.1300 & 37.2922 & 12.1300 & 37.2922 \\
women & 12.2846 & 37.2372 & 12.2846 & 37.2372 \\
Average & 12.2525 & 37.2486 & 12.2525 & 37.2486 \\
\hline
\end{tabular}




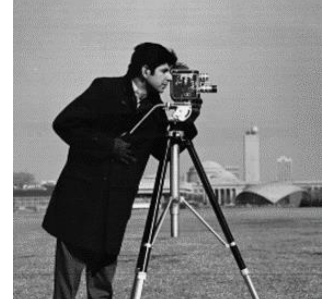

Cameraman DWT-DTT

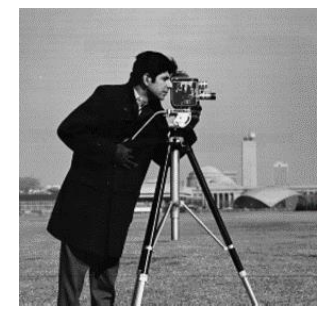

Cameraman DWT-DCT

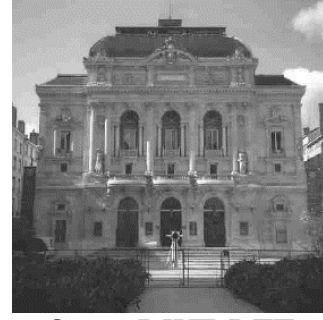

Opera DWT-DTT

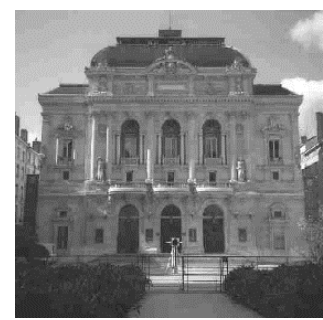

Opera DWT-DCT

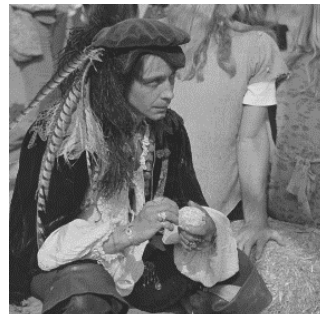

Pirate DWT-DTT

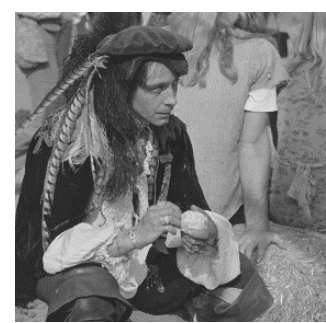

Pirate DWT-DCT

Figure 6. Comparison of the watermarked image using DWT-DTT and DWT-DCT

The PSNR and MSE values are displayed in Table 1 are quite surprising because both values are very identic even exactly the same when rounded into four numbers behind the commas. Where the alpha value used in this study is 30 . This result is real and not intentional, the test has also been done repeatedly and showed the same results. At the stage of extraction used normalize cross-correlation (NCC) [34]. Where the value of NCC can be calculated by (11). NCC serves to calculate the correlation of watermark image with extraction watermark image. Figure 7 shows the NCC values of watermark extraction on each method.

$$
N C C=\frac{\sum_{i=1}^{m} \sum_{j=1}^{n} w(i, j) x w \prime(i, j)}{\sqrt{\sum_{i=1}^{m} \sum_{j=1}^{n} w(i, j)} \sqrt{\sum_{i=1}^{m} \sum_{j=1}^{n} w \prime(i, j)}}
$$

where:

$w(i, j)=$ pixel value from original watermark image

$w^{\prime}(i, j)=$ pixel value from extracted watermark image

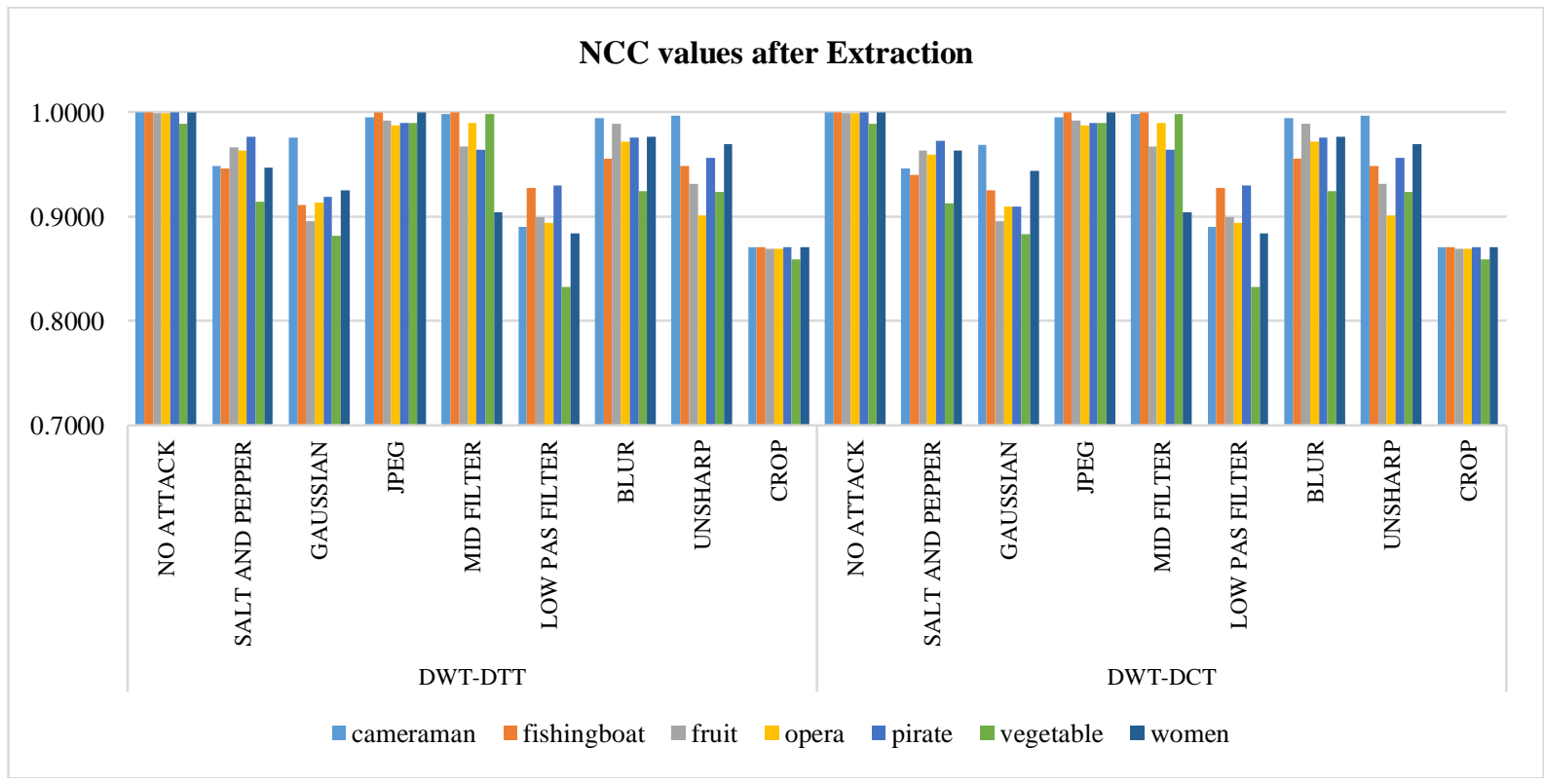

Figure 7. NCC values Chart 
Based on the observations in Figure 7 and Figure 8 it appears that both methods are also equally strong in the robustness aspect. It is very differentiated only at the time needed for the calculation process both in the process of watermark insertion, as well as watermark extraction, where the results can be seen in Table 2. Measurement of time required using the tic toc function on MATLAB and with the same hardware.

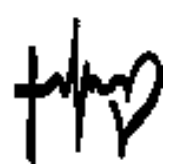

(a)

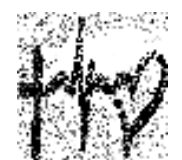

(b)

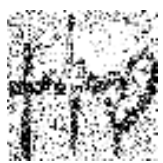

(c)

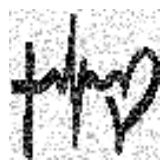

(d)

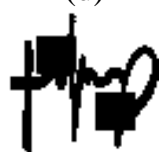

(i)

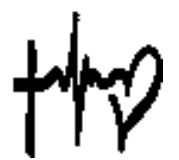

(j)

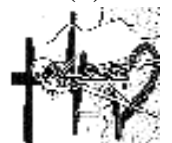

(g)

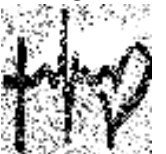

$(\mathrm{k})$

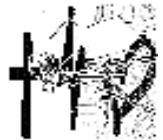

(h)

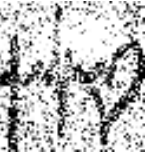

(l)

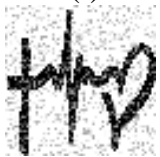

(m)

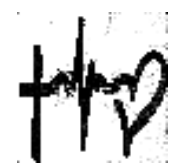

(e)

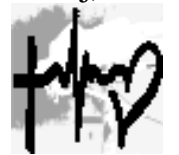

(o)

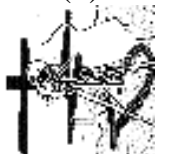

(p)

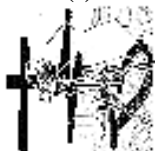

(q)

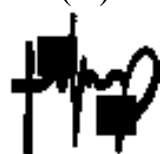

(r)

Figure 8. Sample Extracted Watermark from Cameraman Image (a-i) using DWT-DTT; (j-r) using DWTDCT $\{(\mathrm{a}, \mathrm{j})$ no attack; (b,k) salt and pepper; (c,l) Gaussian noise; (d,m) JPEG compression; (e,n) mid filter; $(\mathrm{f}, \mathrm{o})$ low pass filter; $(\mathrm{g}, \mathrm{p})$ blur; $(\mathrm{k}, \mathrm{q})$ sharpening; (i,r)cropping $\}$

Table 2. Comparative time took

\begin{tabular}{ccccc}
\hline \multirow{2}{*}{ Image } & \multicolumn{2}{c}{$\begin{array}{c}\text { DWT-DTT (in a second) } \\
\text { Embed }\end{array}$} & Extract & \multicolumn{2}{c}{$\begin{array}{c}\text { DWT-DCT (in a second) } \\
\text { Embed }\end{array}$} \\
\hline cameraman & 0.6454 & 0.4174 & 1.8706 & 1.1138 \\
fishing boat & 0.6456 & 0.4108 & 1.9918 & 1.1003 \\
fruit & 0.6497 & 0.4077 & 1.8460 & 1.2029 \\
opera & 0.6386 & 0.4134 & 1.8142 & 1.1403 \\
pirate & 0.6420 & 0.4180 & 1.8460 & 1.1225 \\
vegetable & 0.6889 & 0.4020 & 1.8280 & 1.1059 \\
women & 0.6519 & 0.4076 & 1.8262 & 1.1329 \\
Average & 0.6517 & 0.4110 & 1.8604 & 1.1312 \\
\hline
\end{tabular}

\section{CONCLUSION}

Based on the previous studies and simulations results and also performance tests that have been tested in this study, it can be concluded that the performance of DTT and DCT from the aspect of imperceptibility and robustness is completely identical in the non-blind watermarking method when combined with wavelet transformation. But DTT has an advantage in calculation times that are much better bathed with DCT. Then DTT needs to be studied further and developed again because it is potentially as an alternative DCT in image watermarking method.

\section{REFERENCES}

[1] D. R. I. M. Setiadi, "Payload Enhancement on Least Significant Bit Image Steganography Using Edge Area Dilation," Intl J. Electron. Telecommun., vol. 65, pp. 295-300, 2019.

[2] D. R. I. M. Setiadi and J. Jumanto, "An enhanced LSB-Image Steganography Using the Hybrid Canny-Sobel edge detection," Cybern. Inf. Technol., vol. 18, pp. 74-88, 2018.

[3] R. D. Ardy, et al., "Digital image signature using triple protection cryptosystem (RSA, Vigenere, and MD5)," Proceeding of 2017 International Conference on Smart Cities, Automation and Intelligent Computing Systems, ICON-SONICS 2017, 2017. 
[4] F. Ernawan, "Tchebichef image watermarking along the edge using YCoCg-R color space for copyright protection," Int. J. Electr. Comput. Eng., vol. 9, pp. 1850-1860, 2019.

[5] P. S. N, C. S. S. and M. C. S, "Performance analysis of DCT and successive division based digital image watermarking scheme," Indones. J. Electr. Eng. Comput. Sci., vol. 15, pp. 804-813, 2019.

[6] R. Ahuja and S. S. Bedi, "Video Watermarking Scheme based on Candidates I-Frames for Copyright Protection," Indones. J. Electr. Eng. Comput. Sci., vol. 5, pp. 391-400, 2017.

[7] C. Deng, et al., "A local Tchebichef moments-based robust image watermarking," Signal Processing, vol. 89, pp. 1531-1539, 2009.

[8] S. M. Elshoura and D. B. Megherbi, "Analysis of noise sensitivity of Tchebichef and Zernike moments with application to image watermarking," J. Vis. Commun. Image Represent., vol. 24, pp. 567-578, 2013.

[9] D. R. I. M. Setiadi, et al., "Fast and efficient image watermarking algorithm using discrete tchebichef transform," International Conference on Cyber and IT Service Management (CITSM), pp. 1-5, 2017.

[10] C. A. Sari, et al., "Robust and imperceptible image watermarking by DC coefficients using singular value decomposition," International Conference on Electrical Engineering, Computer Science and Informatics (EECSI), 2017.

[11] E. H. Rachmawanto, et al., "Imperceptible and secure image watermarking using DCT and random spread technique," TELKOMNIKA (Telecommunication Comput. Electron. Control., vol. 17, 2019.

[12] W. S. Sari, et al., "A Good Performance OTP encryption image based on DCT-DWT steganography,” Telkomnika (Telecommunication Comput. Electron. Control), vol. 15, pp. 1982-1989, 2017.

[13] K. Hannoun, et al., "A Novel DWT Domain Watermarking Scheme Based On a Discrete-Time Chaotic System," IFAC-PapersOnLine, vol. 51, pp. 50-55, 2018.

[14] M. Moosazadeh and G. Ekbatanifard, "A new DCT-based robust image watermarking method using teachinglearning-Based optimization,” J. Inf. Secur. Appl., vol. 47, pp. 28-38, 2019.

[15] R. A. Alotaibi and L. A. Elrefaei, "Text-image watermarking based on integer wavelet transform (IWT) and discrete cosine transform (DCT)," Appl. Comput. Informatics, 2018.

[16] A. Winarno, et al., "Image watermarking using low wavelet subband based on $8 \times 8$ sub-block DCT," International Seminar on Application for Technology of Information and Communication (iSemantic), pp. 11-15, 2017.

[17] R. Thanki, et al., "Hybrid and blind watermarking scheme in DCuT - RDWT domain," J. Inf. Secur. Appl., vol. 46, pp. 231-249, 2019.

[18] R. K. Arya, et al., "A secure non-blind block based digital image watermarking technique using DWT and DCT," International Conference on Advances in Computing, Communications and Informatics, pp. 2042-2048, 2015.

[19] U. Sudibyo, et al., "A secure image watermarking using Chinese remainder theorem based on haar wavelet transform," Proceedings - 2017 4th International Conference on Information Technology, Computer, and Electrical Engineering, ICITACEE 2017, 2018.

[20] A. Susanto, et al., "Hybrid method using HWT-DCT for image watermarking," 5th International Conference on Cyber and IT Service Management, CITSM 2017, 2017.

[21] P. A. M. Oliveira, et al., "Low-Complexity Image and Video Coding Based on an Approximate Discrete Tchebichef Transform," IEEE Trans. Circuits Syst. Video Technol., vol. 27, pp. 1066-1076, 2017.

[22] C. C. Bello and J. S. R/ Lopez, "Some computational aspects of Tchebichef moments for higher orders," Pattern Recognit. Lett., vol. 112, pp. 332-339, 2018.

[23] F. Ernawan, et al., "An efficient image compression technique using Tchebichef bit allocation," Optik (Stuttg), vol. 148, pp. 106-119, 2017.

[24] H. I. Saleh, “A Fast Block-Pruned 4x4 DTT Algorithm for Image Compression,” Int. J. Comput. Theory Eng., pp. 258-261, 2009.

[25] S. M. Elshoura and D. B. Megherbi, "High capacity blind information hiding schemes using Tchebichef moments," 2010 2nd International Conference on Future Computer and Communication, pp. V1-649-V1-653, 2010.

[26] P. A. M. Oliveira, et al., "A Discrete Tchebichef Transform Approximation for Image and Video Coding," IEEE Signal Process. Lett., vol. 22, pp. 1137-1141, 2015.

[27] B. Xiao, et al., "Lossless image compression based on integer Discrete Tchebichef Transform," Neurocomputing, vol. 214, pp. 587-593, 2016.

[28] I. Batioua, et al., "3D image analysis by separable discrete orthogonal moments based on Krawtchouk and Tchebichef polynomials," Pattern Recognit., vol. 71, pp. 264-277, 2017.

[29] R. K. Senapati, et al., "Image Compression Using Discrete Tchebichef Transform Algorithm," International Conference on Advances in Communication, Network, and Computing, 2010.

[30] A. Setyono, et al., "StegoCrypt method using wavelet transform and one-time pad for secret image delivery," International Conference on Information Technology, Computer, and Electrical Engineering, pp. 203-207, 2017.

[31] V. A. Kumar, et al., "A Hybrid Digital Watermarking Approach Using Wavelets and LSB," Int. J. Electr. Comput. Eng., vol. 7, pp. 2483, 2017.

[32] M. Chi and Z. Yongyong, "A Novel Self-adaptive Discrete Wavelet Transform Digital Watermarking Algorithm," Indones. J. Electr. Eng. Comput. Sci., vol. 11, 2013.

[33] L. Alasafi, et al., "Copyright Protection by Robust Digital Image Watermarking in Unsecured Communication Channels," Indones. J. Electr. Eng. Comput. Sci., vol. 7, pp. 234-249, 2017.

[34] M. N. M. Najih, et al., "An improved secure image hiding technique using PN-sequence based on DCT-OTP," 2017 1st International Conference on Informatics and Computational Sciences (ICICoS), pp. 47-52, 2017. 